\title{
EFFECTS OF ROUGH TOPOGRAPHY IN GNSS-R: A PARAMETRIC STUDY BASED ON A DIGITAL ELEVATION MODEL
}

\author{
Hugo Carreno-Luengo, Guido Luzi, and Michele Crosetto \\ Centre Tecnòlogic de Telecomunicacions de Catalunya (CTTC/CERCA), Castelldefels (Barcelona), Spain \\ Tel. +34 9364529 00, E-mail: hugo.carreno@cttc.cat
}

\begin{abstract}
Understanding the effects of Earth's surface topography in Global Navigation Satellite Systems Reflectometry (GNSS-R) spaceborne data is essential to correct experimental observations so as to achieve accurate Soil Moisture Content (SMC) determination and biomass monitoring. A comprehensive set of observables obtained from Delay Doppler Maps (DDMs) generated on-board Cyclone Global Navigation Satellite Systems (CyGNSS) are evaluated as a function of several topographic parameters derived from a Digital Elevation Model (DEM). Terrain Ruggedness Index (TRI) and profile curvature have a moderate-to-strong impact on CyGNSS-derived trailing edge (TE) and reflectivity $\Gamma$ for low elevation angles $\theta_{\mathrm{e}} \sim[20,30]^{\circ}\left(\mathbf{r}_{-} \mathrm{TRI}_{-} \Gamma \sim \mathbf{- 0 . 3 9}\right.$, r_TRI_TE $\sim 0.39, r_{-} \_$curv $\_\Gamma \sim 0.41, r_{-}$curv_ $\left.T E \sim-0.38\right)$. On the other hand, local slopes have an influence on $\Gamma$ and TE for a constant curvature level, while both observables are independent of the slopes for a constant TRI.
\end{abstract}

Index Terms - GNSS-R, CyGNSS, topography, digital elevation model, elevation angle, soil moisture content

\section{INTRODUCTION}

Topography is a key element in several processes such as hydrology, climatology, geography, and ecology. On the other hand, it is well known that topography distorts monostatic radar signatures. However, it remains uncertain up to what extend topography influences GNSS-R data and how these effects can be corrected [1-3]. This aspect remains a bottleneck in the community.

GNSS-R missions are aimed to revolutionize several scientific disciplines thanks to their passive multistatic radar properties [4]. The application of GNSS-R over land surfaces is becoming of increasing interest, however only a reduced number of studies have been performed using spaceborne data. On the other hand, the relief-induced distorsions remain uncorrected.

The main purpose of the present investigation is to further understand the effects of rough topography using data from the CyGNSS eight-microsatellites Global
Positioning System (GPS) C/A code-based constellation $[5,6]$. The counterpart of the "radar pulsewidth" in CyGNSS is $~ 300 \mathrm{~m}$ ( $1 \mathrm{C} / \mathrm{A}$ code chip). As such, the reflected DDMs [7] are sensitivite to the relative orientation of the scattering surfaces, but also to the vertical relief distribution. At L-band $(\lambda \sim 20 \mathrm{~cm})$, the scattering over areas with rough topography should be totally diffuse, and the statistics of the reflected signals should be similar to that of speckle noise.

\section{EFFECTS OF TOPOGRAPHY IN DELAY DOPPLER MAPS}

Over regions without rough topography, DDMs can be expressed as follows:

$$
\mathrm{Y}\left(\tau, \mathrm{f}_{\mathrm{D}}\right)=\left|\chi\left(\tau, \mathrm{f}_{\mathrm{D}}\right)\right|^{2} * *\left|\sigma^{0}\left(\tau, \mathrm{f}_{\mathrm{D}}\right)\right|^{2},
$$

where $\chi$ is the Woodward Ambiguity Function (WAF), $\sigma^{0}$ is the bistatic scattering coefficient, $\tau$ is the delay of the signal from the transmitter to the receiver, $f_{D}$ is the Doppler shift of the reflected electromagnetic signal, and $* *$ is the 2-D convolution in both domains, delay and Doppler.

The WAF can be defined as follows:

$$
\left|\chi\left(\tau, \mathrm{f}_{\mathrm{D}}\right)\right|=\Lambda(\tau) \mathrm{S}\left(\mathrm{f}_{\mathrm{D}}\right),
$$

where $\Lambda$ is the autocorrelation function of the PseudoRandom Noise (PRN) codes and $S$ is the system impulse response in the frequency domain. The WAF defines the delay and Doppler selectivity of the coherent radar [7].

Regions with rough topography are characterized with a RMS of the surface height variation higher than the length of the GNSS-code under study. The effects of rough topography in the DDMs can be understood as:

$$
\mathrm{Y}_{\text {top }}\left(\tau, \mathrm{f}_{\mathrm{D}}\right)=\mathrm{Y}\left(\tau, \mathrm{f}_{\mathrm{D}}\right) * * \xi\left(\tau, \mathrm{f}_{\mathrm{D}}\right),
$$

where the function $\xi$ represents the mean density of scattering points as a function of the delay and the Doppler shift.

$\xi$ depends on the local topography but also on the GNSS satellites elevation and azimuth angles, the relative velocity of the transmitter and the receiver, etc. 


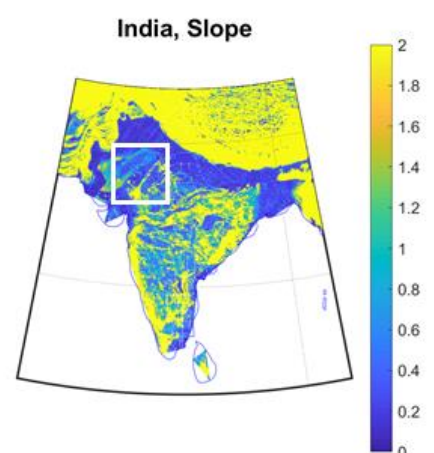

(a)

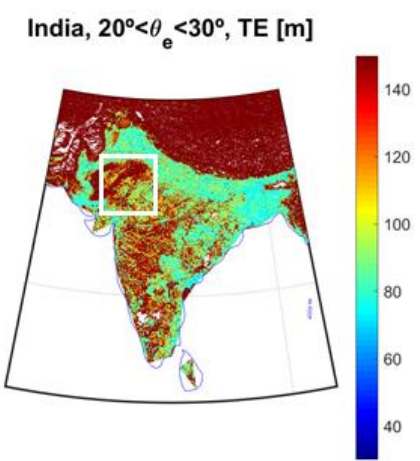

(e)

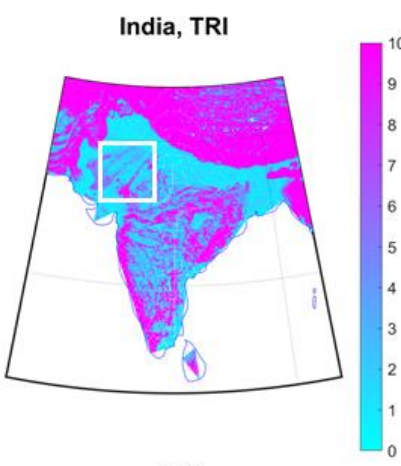

(b)

India, $80^{\circ}<\theta_{\mathrm{e}}<90^{\circ}$, TE [m]

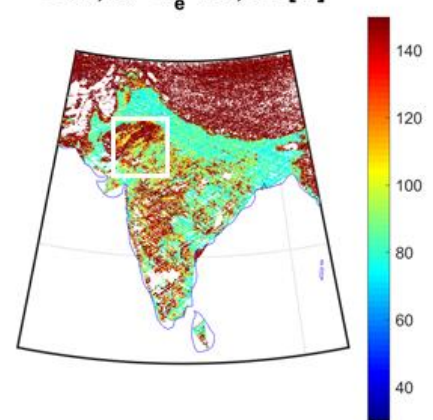

(f)

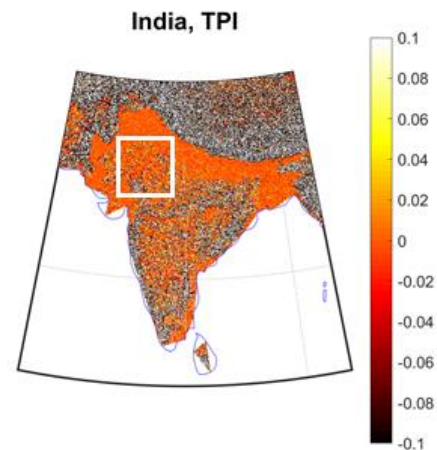

(c)

India, $20^{\circ}<\theta_{\mathrm{e}}<30^{\circ}, \Gamma[\mathrm{dB}]$

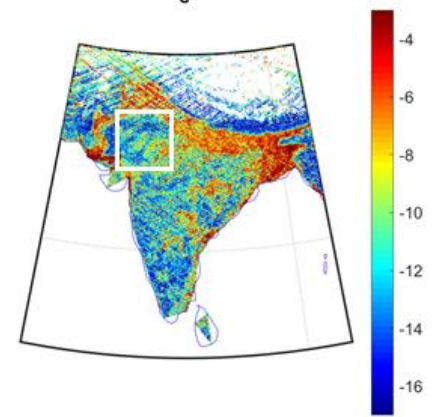

(g)

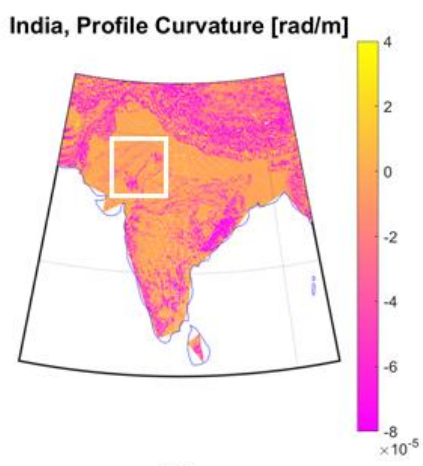

(d)

India, $80^{\circ}<\theta_{\mathrm{e}}<90^{\circ}, \Gamma[\mathrm{dB}]$

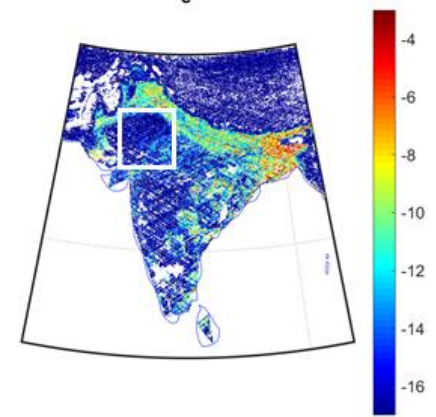

(h)

Fig. 1. Overview of a subset of topographic parameters derived from the $250 \mathrm{~m}$ GMTED2010 [8]: (a) Slope, (b) Topographic Ruggedness Index (TRI), (c) Topographic Position Index (TPI), and (d) Profile Curvature. 6 months (01/08/2018 to 31/01/2019) of CyGNSS-derived trailing edge (e),(f) TE and reflectivity (g),(h) $\Gamma$ at $\theta_{\mathrm{e}} \sim[20,30]^{\circ}(\mathrm{e}),(\mathrm{g})$ and $\theta_{\mathrm{e}} \sim[80,90]^{\circ}(\mathrm{f}),(\mathrm{h})$. White boxes hightlight the specific target area under study: Lat. $=\left[22,29{ }^{\circ}\right.$, Lon. $=[70,77]^{\circ}$.

\section{METHODOLOGY}

\subsection{TOPOGRAPHIC PARAMETERS}

Topographic heterogeneity is described as the variability of elevations within an area. Topography is an important parameter that disturbs $\mathrm{Y}\left(\tau, \mathrm{f}_{\mathrm{D}}\right)$ because local surface slopes modify the scattering coefficient. In this work, the DEM products of $250 \mathrm{~m}$ from the Global Multi-resolution Terrain Elevation Data 2010 (GMTED2010) are used to derive the following topographic descriptors using a $3 \times 3$ cell moving window [8]:

- Terrain Ruggedness Index (TRI): Mean of the absolute differences in elevation between a focal cell and its 8 surrounding cells. It quantifies the total elevation change across the $3 \times 3$ cells. Flat areas have a value of zero and mountain areas with steep ridges have positive values up $\sim 2000$ over the Hymalayan area.

- Topographic Position Index (TPI): Difference between the elevation of a focal cell and the mean of its 8 surrounding cells. Positive and negative values correspond to ridges and valleys, respectively.

- Slope: Rate of change of elevation in magnitude for the steepest descet vector. It is computed as the first derivative of the surface elevation. Slope and curvature serve as useful input variables in erosion and hydrological models.

- Profile curvature: Curvature is based on the change of slope in a particular direction. Profile curvature measures the change rate of slope along a flow line. It affects the acceleration or deceleration of surface flow along the surface and thus it influences erosion and deposition of soils. The unit of curvature is $\mathrm{rad} / \mathrm{m}$. Positive and negative values indicate convex and concave surfaces, respectively. It is computed as the second derivative of the elevation.

These parameters provide a better understanding of the properties of the terrain than raw DEM data. TRI, TPI 


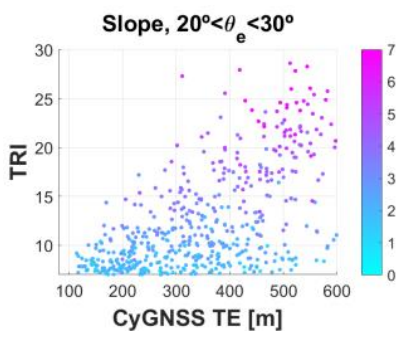

(a)

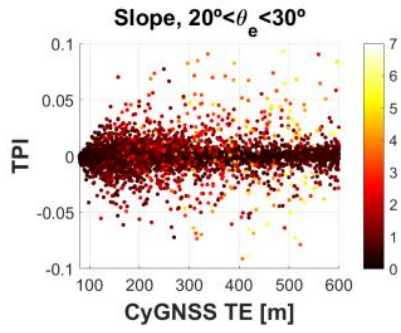

(e)

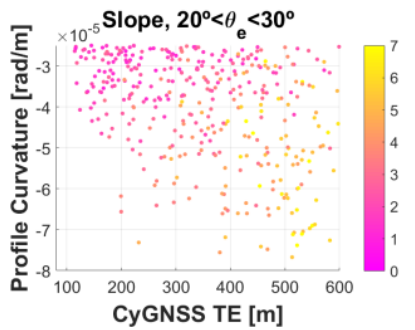

(i)

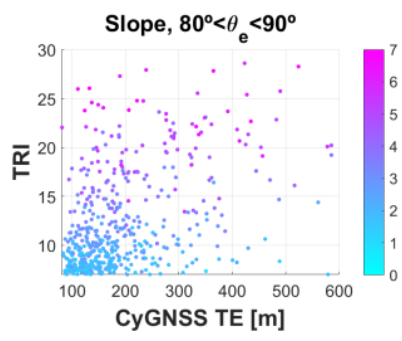

(b)

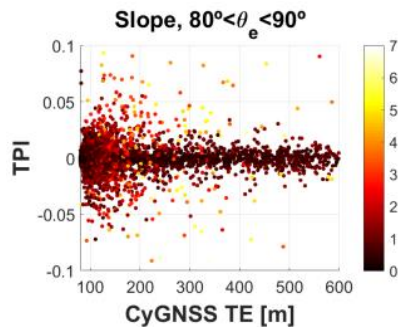

(f)

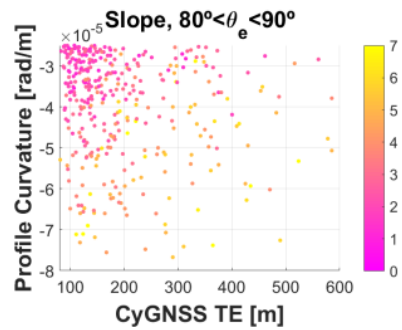

(j)

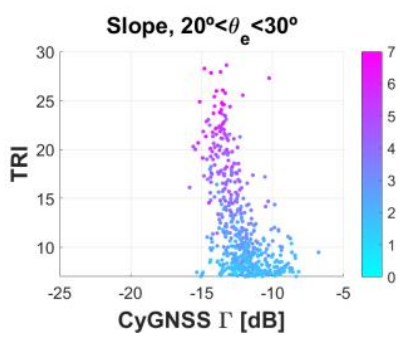

(c)

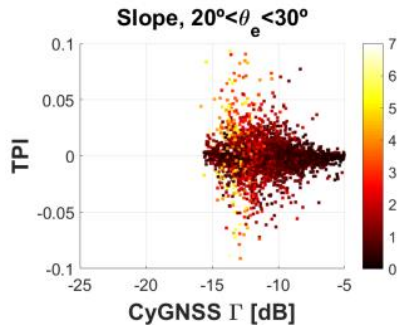

(g)

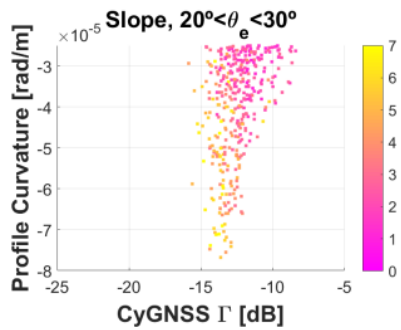

(k)

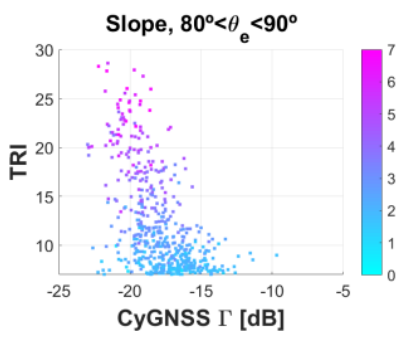

(d)

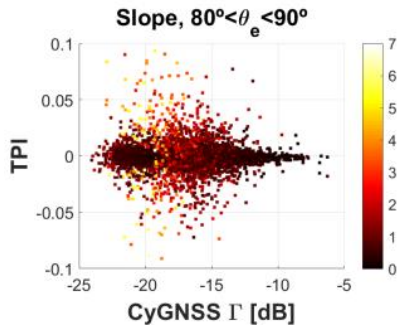

(h)

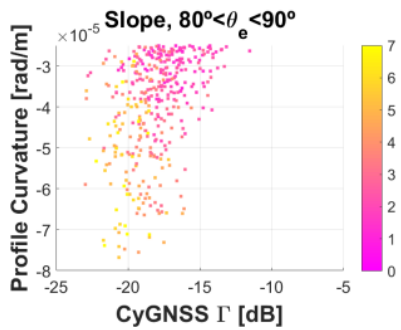

(l)

Fig. 2. Scatter plots: (a)-(d) Terrain Ruggedness Index (TRI) with Trailing Edge ( TE ) and reflectivity $\Gamma$ for elevation angles of (a),(c) $\theta_{\mathrm{e}} \sim[20,30]^{\circ}$ and (b),(d) $\theta_{\mathrm{e}} \sim[80,90]^{\circ}$. (e)-(h) Terrain Position Index (TPI) with TE and reflectivity $\Gamma$ for elevation angles of (e),(g) $\theta_{\mathrm{e}} \sim[20,30]^{\circ}$ and (f), (h) $\theta_{\mathrm{e}} \sim$ $[80,90]^{\circ}$. (i)-(l) Profile curvature with TE and reflectivity $\Gamma$ for elevation angles of (i),(k) $\theta_{\mathrm{e}} \sim[20,30]^{\mathrm{o}}$ and (j),(l) $\theta_{\mathrm{e}} \sim[80,90]^{\circ}$. All plots are displayed as a function of the slope. Target area over India: Lat. $=[22,29]^{\circ}$, Lon. $=[70,77]^{\circ}$.

provide different descriptions of the topographic heterogeneity. The curvature is described based on changes of slope.

\subsection{DELAY DOPPLER MAPS PARAMETERS}

CyGNSS Level 2.1 Science Data Record [4,5] is selected for this study. TE and $\Gamma$ are defined and computed following a similar approach as in a companion paper [9]. Here, this work is focused on a deeper underdtaing of the effects of rough topography in the GNSS-R observables.

\section{A CASE STUDY OVER INDIA: METHODS AND PRELIMINARY RESULTS}

6-month of CyGNSS data (01/08/2018 to 31/01/2019) are averaged using a $0.1^{\circ} \mathrm{x} 0.1^{\circ}$ latitude/longitude grid with a moving window of $0.2^{\circ}$ in steps of $0.1^{\circ}$. DDMs are classified into different groups according to different ranges of the satellites' elevation angle $\theta_{\mathrm{e}} \sim[20,30]^{\circ}$, and $\theta_{\mathrm{e}} \sim[80,90]^{\circ}$.
Raw topographic variables are first aggregated using the mean at a spatial resolution of $\sim 5 \mathrm{~km}$ [10]. Then, a similar moving averging window is applied. This strategy enables the analysis using auxiliary data from different sensors with a variety of spatial resolutions.

Fig. 1 shows the analysis over India with low-to-high topographic heterogeneity levels, including the Hymalayas region. TE significantly increases with higher values of TRI, TPI, slopes, and profile curvature [Figs. 1(e),(f)] because surface scattering becomes more diffuse for higher values of terrain heterogeneity variables. On the other hand, $\Gamma$ increases over regions with lower topographic heterogeneity [Figs. 1(g),(h)] because surface scattering is more specular over a less convex terrain, which in turn increases the water accumulation rate of the surface.

The impact of the elevation angle $\theta_{\mathrm{e}}$ is also evaluated. At a regional scale [Fig. 1], both $\mathrm{TE}$ and $\Gamma$ increase for 
TABLE I: Statistical analysis of the linear regressions between CyGNSSderived scientific observables and the topographic heterogeneity parameters for $\theta_{\mathrm{e}} \sim[20,30]^{\circ}$.

\begin{tabular}{ccccccc}
\hline DEM & TRI & TRI & TPI & TPI & P.Curv. & P.Curv. \\
\hline CyGNSS & TE & $\Gamma$ & TE & $\Gamma$ & TE & $\Gamma$ \\
\hline r & 0.39 & -0.39 & 0.04 & -0.06 & -0.38 & 0.41 \\
\hline Slope & 0.02 & -1.84 & $4 * 10^{-6}$ & $-3 * 10^{-4}$ & $-3 * 10^{-8}$ & $4 * 10^{-6}$ \\
\hline RMSE & 4.32 & 4.48 & 0.0143 & 0.0143 & $1 * 10^{-5}$ & $1 * 10^{-5}$ \\
\hline
\end{tabular}

TABLE II: Statistical analysis of the linear regressions between CyGNSSderived scientific observables and the topographic heterogeneity parameters for $\theta_{\mathrm{e}} \sim[80,90]^{\circ}$.

\begin{tabular}{ccccccc}
\hline DEM & TRI & TRI & TPI & TPI & P.Curv. & P.Curv. \\
\hline CyGNSS & TE & $\Gamma$ & TE & $\Gamma$ & TE & $\Gamma$ \\
\hline r & 0.23 & -0.29 & 0.02 & -0.03 & -0.21 & 0.32 \\
\hline Slope & 0.02 & -1.39 & $2 * 10^{-6}$ & $-1 * 10^{-4}$ & $-2 * 10^{-6}$ & $2 * 10^{-6}$ \\
\hline RMSE & 4.73 & 4.35 & 0.0142 & 0.0142 & $1 * 10^{-5}$ & $1.2 * 10^{-5}$ \\
\hline
\end{tabular}

lower angles $\theta_{\mathrm{e}} \sim[20,30]^{\circ}$. This point deserves deeper investigation. Fig. 2 shows the scatter plots of the selected topograpraphic variables with $\mathrm{TE}$ and $\Gamma$ over a smaller target area over Northwest India (Lat. $=[22,29]^{\circ}$, Lon. $=$ $[70,77]^{\circ}$ ) to frame potential uncertainties due to vegetation and potential errors in the determination of the specular reflection points over the Hymalayas areas. TE and $\Gamma$ are more correlated with TRI and profile curvature at $\theta_{\mathrm{e}} \sim[20$, $30]^{\circ}$ (r_tRL_ $\Gamma \sim-0.39, r_{-}$TRL_TE $\sim 0.39, r_{-}$curv $-\Gamma \sim 0.41$, $\mathrm{r}_{- \text {curv }}$ TE $\left.\sim-0.38\right)$ than at $\theta_{\mathrm{e}} \sim[80,90]^{\circ}\left(\mathrm{r}_{-}\right.$TRI_ $\Gamma \sim-0.29$, r_TRI_TE $\sim 0.23, \mathrm{r}_{-}$curv_ $\Gamma \sim 0.32, \mathrm{r}_{-}$curv_ TE $\left.\sim-21\right)$. The impact of TPI is almost negligigle [Figs. 2 (e)-(h) and Tables I\&II].

Local slopes cause the scattering area to be different from that calculated using a flat Earth assumption. Thus, they are a key-parameter in GNSS-R studies over land surfaces. In the case of arbitrary terrain slopes, the phase difference between two surface-scattered electromagnetic signals become uniformely random because the path length difference is several wavelengths. As such, on average, the resulting scattered power consists on the sum of the powers in the individuals waves.

Slope is the selected parameter to evaluate the relationship of the selected scientific observables with the selected heterogeneity variables. It is found that slopes increases with higher TRI [Figs. 2(a)-(d)] and lower curvature values [Figs. 2 (i)-(l)]. For the same curvature level, higher slopes belong to an increment of TE [Figs. 2(i),(j)] and a reduction of $\Gamma$ [Figs. 2(k),(l)]. On the other hand, both CyGNSS observables appear to be independent of slopes for the same TRI value [Figs. 2(a)-(d)].

\section{CONCLUSIONS}

Preliminary results show that spaceborne DDMs' parameters such as trailing edge TE and reflectivity $\Gamma$ have a moderate-to-strong influence of the topographic heterogeneity and curvature DEM-derived parameters. This impact is higher at $\theta_{\mathrm{e}} \sim[20,30]^{\circ}$ than at $\theta_{\mathrm{e}} \sim[80,90]^{\circ}$. Accurate retrieval of geophysical parameters such as Soil Moisture Content (SMC) and biomass requires to calibrate the collected Earth-reflected signals when there are mountains over the glistening zone. The effects could be up to the order of $\sim 5 \mathrm{~dB}$ and several tens/hundreds of meters over India.

\section{REFERENCES}

[1] H. Park, A. Camps, D. Pascual, A. Alonso-Arroyo, J. Querol, and R. Onrubia, "Improvement of PAU/PARIS end-to-end performance simulator (P2EPS): land scattering including topography," in Proceedings of the IEEE International Geoscience and Remote Sensing Symposium, pp. 56075610, Beijing, China, 10-15 July 2016.

[2] H. Carreno-Luengo, S. T. Lowe, C. Zuffada, S. Esterhuizen, and S. Oveisgharan, "Spaceborne GNSS-R from the SMAP mission: first assessment of polarimetric scatterometry over land and cryosphere," MDPI Remote Sensing, vol. 9. no. 361, pp. 362, Apr. 2017.

[3] H. Park, A. Camps, D. Pascual, J. Querol, and R. Onrubia, "Spaceborne GNSS-R end-to-end simulator: topography and vegetation effects," in Proceedings of the IEEE International Geoscience and Remote Sensing Symposium, pp. 3157-3160, Valencia, Spain, 22-27 July 2018.

[4] E. Cardellach \& J. Wickert et al., GNSS Transpolar Earth Reflectometry exploriNg System (G-TERN): mission concept," IEEE Transactions of Geoscience and Remote Sensing, vol. 6, pp. 13980-14018, Mar. 2018.

[5] C. Ruf et al., "New ocean winds satellite mission to probe hurricanes and tropical convection,” Bull. Amer. Meteorol. Soc., vol. 97, pp. 385-395, 2015, doi: 10.1175/BAMS-D-14-00218.1.

[6] CYGNSS. 2017. CYGNSS Level 1 Science Data Record. Ver. 2.1. PO.DAAC, CA, USA. [Online]. Available http://dx.doi.org/10.5067/CYGNS-L1X20 (23/04/2019).

[7] V.U. Zavorotny, S. Gleason, E. Cardellach, and A. Camps, "Tutorial on remote sensing using GNSS bistatic radar of opportunity," IEEE Geoscience and Remote Sensing Magazine, vol. 2, no. 4, pp. 8-45, Dec. 2014.

[8] G. Amatulli, S. Domisch, M.-N. Tuanmu, B. Parmentire, A. Ranipeta, J. Malczyk, and W. Jetz, "A suite of global cross-scale topographic variables for environmental and biodiversity modelling," Nature Scientific data, Mar. 2018. DOI: 10.1038/sdata.2018.40

[9] H. Carreno-Luengo, G. Luzi, and M. Crosetto, "Biomass estimation over tropical rainforests using GNSS-R on-board the CyGNSS microsatellites constellation," in Proceedings of the IEEE International Geoscience and Remote Sensing Symposium, Yokohama, Japan, 28 July-2 August 2019.

[10] EarthEnv dataset. [Online]. Available https://www.earthenv.org/topography (02/03/2019).

\section{ACKOWLEDGEMENTS}

This research was carried out with the support grant of a Juan de la Cierva research fellowship from the Spanish Ministerio de Economia, Industria y Competitividad (MINECO), reference FJCI2016-29356. Travel expenses were partially covered by the Postdoctoral Award sponsored by the open access journal Remote Sensing published by MDPI. 